\title{
Identidad cultural de los jóvenes de Siuna, Costa Caribe Norte de Nicaragua
}

\author{
Dayling del Socorro Tórrez Jarquín' \\ Anielka Ellieth Urbina Larios² \\ Lilia del Rosario Montoya Leal ${ }^{3}$
}

\section{Resumen}

n este estudio se ha analizado la identidad cultural de los jóvenes de la ciudad de Siuna. Se trata de una Einvestigación cualitativa con un enfoque etnometodológico, donde los datos obtenidos son el resultado de haber suministrado diferentes instrumentos de investigación como la entrevista y la observación a padres y madres de familia, jóvenes y personas mayores de 50 años. Los resultados muestran factores que inciden en la identidad cultural de las personas jóvenes como sociales, culturales, tecnológicos y económicos. Así mismo, se han descrito las vivencias de la identidad cultural, a través de la relación con las amistades, la música que escuchan, la apariencia, modos de actuar, el contenido audiovisual a los cuales tienen acceso y la práctica de valores desde la familia. Estas manifestaciones tienen carácter simbólico, material y no se puede generalizar de la misma manera a toda la juventud del municipio, pues el contexto y las condiciones en las que les toca vivir son diferentes. Para concluir, se proponen acciones tales como charlas a padres y madres de familia, implementar un plan de sensibilización, campañas de incidencia social dirigidas a jóvenes para promover el rescate de valores y prácticas culturales.

Palabras clave: Identidad cultural; juventud; factores de incidencia; vivencias.

\section{Abstract}

In this study, the cultural identity of the young people of the city of Siuna has been analyzed. It is a qualitative research with an ethnomethodological approach, where the obtained data are the result of having provided different research instruments such as interview and observation to parents, young people and people over 50 years. The results show factors affecting the cultural identity of young people as social, cultural, technological and economic. Likewise, the experiences of cultural identity have been described through the relationship with the friends, the music they listen to, the appearance, ways of acting, the audiovisual content which they have access and the practice of values from the family. These manifestations are symbolic, material and cannot be generalized in the same way to all the youth of the municipality, because the context and the conditions in which they have to live are different. In conclusion, actions such as lectures to parents are proposed, implement an awareness-raising plan, social advocacy campaigns aimed at young people to promote the rescue of cultural values and practices.

Keywords: Cultural identity; youth; Incidence factors; Experiences.

\footnotetext{
1 Licenciada en Ciencias Sociales con Mención en Desarrollo Local. Asistente de Vicerrectora de la Universidad de las Regiones Autónomas de la Costa Caribe Nicaragüense-Recinto Universitario Las Minas. Correo: daylingt@yahoo.es

2 Licenciada en Ciencias Sociales con Mención en Desarrollo Local. Correo: anielkaurbina2014@hotmail.com

3 Magister en Educación y Desarrollo. Coordinadora Área de Humanidades de la Universidad de las Regiones Autónomas de la Costa Caribe Nicaragüense-Recinto Universitario Las Minas. Correo: lilialeal14@yahoo.com
}

Recibido: 21/04/2017 Aprobado: 12/06/2017 


\section{Introducción}

Esta investigación trató de analizar la identidad cultural de los jóvenes de la ciudad de Siuna, teniendo en cuenta que la juventud está viviendo una crisis de valores en la actualidad, tanto morales como culturales, identificándose con elementos culturales que pertenecen a un mundo que cada vez más homogéneo en el contexto de globalización. Asimismo, hablar de identidad cultural se refiere a los valores y elementos de una cultura con la cual nos identificamos y de acuerdo a ello nos fijamos manera de expresar y comportarnos.

En América Latina, las identidades culturales y sus nuevas formas de hacer y de pensar, están definiendo los rumbos de las sociedades y estas naciones. Las identidades y culturas tradicionales como las campesinas, indígenas y negras, resisten y construyen nuevas alternativas a la dominación de la globalización cultural, lo que ha impedido la introducción automática de otras culturas foráneas por su capacidad de defender lo propio, lo autóctono de esta tierra (Sánchez, 2012). En Nicaragua se visualizan la existencia de comunidades indígenas, afrodescendientes y mestizas, lo que se convierte en una nación multicultural y plurilingüe (UCA, 2013).

En la Costa Caribe Nicaragüense siempre se han desarrollado procesos muy diferentes al resto del país, por poseer gran parte de la diversidad cultural del país y vivir en contextos tanto geográfico como culturalmente diferentes. Sin embargo, se han excluido. En el municipio de Siuna desde las nuevas tecnologías como los teléfonos móviles, el acceso a la televisión por cable y la internet, los jóvenes muestran comportamientos y actitudes que imitan a sociedades de Europa occidental y norteamericana (UCA, 2013).

Aunque, se puede hablar de adaptación de las generaciones, es evidente que en ese proceso se dejan de practicar características propias de nuestra cultura, esto debido a factores tanto internos como externos en nuestra sociedad. Abordar este tema, resultó muy interesante para el ámbito académico y social, permitió contextualizar cómo se vivencia la identidad cultural de la juventud desde los espacios en que interactúan, tanto actitudes como comportamientos, conocer que influye en ello y los factores inciden en la transformación o el cambio de una generación a otra.

\section{Revisión de literatura}

La identidad cultural es el conjunto de medios por los cuales un individuo se describe a sí mismo (Ramírez, 1993). Es decir, la identidad que comparte un grupo o una población, implica un sentido personal de lealtad. Los miembros de un grupo étnico muestran su identidad por medio de rasgos culturales que pueden cambiar con el tiempo, esta define la identificación a una comunidad y a su forma de vida (Ramírez, 1993, p. 8).

En este sentido, existe una diversidad de factores por los cuales la identidad cultural puede ser cambiante, muchas veces se opta por la que nos hace encajar según las diferencias etarias. Dentro de los factores que influyen en la identidad cultural de las personas se abordaran cuatro clasificaciones: sociales, culturales, tecnológicos y económicos. De los teóricos de la identidad étnica, nos interesa especialmente la importancia que otorgan al contexto donde se desarrolla la identidad cultural y a las relaciones de poder que se dan entre el grupo dominante o mayoritario y los grupos dominados o minorías. Esta corriente defiende que la formación de la identidad cultural está condicionada por varios factores, uno de ellos es el contexto físico donde se lleva a cabo la socialización del individuo (Rascon, 2006, p. 44).

León (2013) manifiesta que día a día somos testigos de los procesos de aculturación en diferentes niveles y diferentes espacios, durante este proceso existe un intercambio de elementos culturales entre distintos grupos sociales, que genera la pérdida de la identidad cultural de los pueblos indígenas (aculturación). Uno de los principales factores que consideramos está en la cima y causa efectos considerables en la formación de identidad en las sociedades y principalmente en la juventud, es la globalización. "La globalización ha producido, nuevas realidades en el concierto internacional, así como transformaciones en las relaciones socio-culturales mundiales, (Sánchez,2012, p. 1).

Para Guerrero (2002) entre los elementos que conforman las cultura están la espacialidad, en este sentido refiere que toda manifestación de la cultura no se produce en el vacío, sino en un espacio concreto, se hace referencia al espacio, universo, escenario, lugar, en donde se produce o realiza un determinado hecho social (pp. 30-63). Entre otros significativos destaca el idioma, las artes, sistema de salud, tradición oral y literatura, arquitectura, 
vestimenta, alimentación, actividades lúdicas (Guerrero, 2002, pp.30-63).

En este sentido, las manifestaciones de las prácticas sociales de los jóvenes, por lo general no son una sola forma de expresar esa "cultura juvenil", sino que es un híbrido cultural, que utilizan los jóvenes en búsqueda de su propia identidad. Podemos reafirmar que ante la complejidad que enfrentan los jóvenes con esta suerte de modernización de las industrias culturales, se da un "creciente tráfico entre culturas que origina la globalización, lo que indica que la desaparición del vínculo entre cultura y lugar viene acompañada por un entrelazamiento de estas prácticas culturales desarraigadas, que producen nuevas y complejas formas híbridas de cultura" (Ramírez et al., 1993, p.2)

Las acciones a tomar en cuenta tienen que ver con el fortalecimiento de la comunicación en la familia, entre padres, madres de familia con sus hijos e hijas, así mismo reforzar la convivencia entre los miembros de este grupo. Gómez \& Suárez (2006) resaltan que la familia y los adultos que representan un papel importante en la infancia.

Promover actividades que permitan a la juventud identificarse con valores y prácticas culturales. Desde la juventud, se puede promover tipos de propuestas entre las que destacamos las siguientes: Incentivar los talentos artísticos y culturales de adolescentes y jóvenes costeños. Creación de centros culturales donde los adolescentes y jóvenes afro descendientes puedan desarrollar sus habilidades artísticas. Coordinar con las alcaldías y gobiernos regionales la creación de un fondo especial para la promoción y rescate de la cultura. Bibliotecas municipales con documentación que contenga información de la historia y cultura de la Costa Caribe.

\section{Materiales y métodos}

Esta investigación es cualitativa porque es una actividad sistemática orientada a la comprensión en profundidad de fenómenos educativos y sociales a la transformación de prácticas y escenarios socioeducativos a la toma de decisiones y también hacia el descubrimiento y desarrollo de un cuerpo organizado de conocimiento (Sandín, 2003, p. 123). En este sentido, esta investigación trata de analizar la identidad cultural de los jóvenes de la ciudad de Siuna de la Costa Caribe Norte de Nicaragua.
Por consiguiente, esta investigación tiene un enfoque etnometodológico porque pretende describir el mundo social tal y como se está continuamente construyendo, emergiendo como realidad objetiva, ordenada, inteligible y familiar. En este sentido, los participantes en esta investigación fueron padres y madres de familia, jóvenes y personas mayores de 50 años. En este contexto se aplicaron entrevista y la observación participativa. Se destaca que el análisis y procesamiento de la información a través de una matriz y los resultados permitieron la discusión con el abordaje teórico.

\section{Resultados y discusión}

\section{Factores que inciden en la formación de la identidad cultural de jóvenes}

Tanto padres, madres y jóvenes manifiestan que la familia es un elemento crucial en la formación de la identidad, pues son el primer contacto que tiene el ser humano al nacer y crecer, es a través de la figura paterna, materna o en algunos casos de los abuelos, que adquiere o aprende actitudes y comportamientos, esto mediante la instrucción de valores, costumbres y tradiciones.

La familia desempeña un papel importante en la formación de la identidad de las personas, en este espacio social se inculcan valores como el respeto, la responsabilidad, la honestidad, el amor, la tolerancia; los cuales, según el grupo de padres y madres entrevistadas, hoy en día están en crisis: "la juventud se muestra cada día más rebelde". Esto concuerda con lo expresado por Gómez (2009), para quien muchos de los principios básicos del comportamiento humano, han sido sustituidos por valores cambiantes en las familias que se apoyan en argumentos de modernidad, ligados al desarrollo científico, técnico y a los cambios sociales.

Así mismo las madres y lo reconocen la influencia que tienen las amistades en la personalidad y comportamiento de los jóvenes, comparten más tiempo con sus grupos etarios que con la familia, hay más confianza para contarse las cosas que les pasan, los problemas, se aceptan entre sí y se comprenden. Se relaciona con De la Hoz (2011), cuando expresa que los jóvenes rechazan la sociedad establecida, entendiendo de un modo totalmente opuesto a los adultos las grandes cuestiones de la vida. Además, los grupos de amigos son una influencia en el cambio de comportamiento de los jóvenes, divulgan la imagen del "joven típico" y los 
que no se ajustan a ella empiezan a notar que es como si les faltase algo.

Respecto a la religión, madres y padres de familia manifestaron que, en la actualidad, a la juventud no le gusta nada que tenga que ver con lo religioso, se encuentran atraídos hacia otras actividades que para ellos son más "divertidas". Manifiestan que en su generación era exigido ir a misa todos los domingos, o al culto todos los días, sentarse a orar en familia, rezar el rosario, el temor a Dios y cumplir con los mandamientos.

Por su parte los jóvenes expresan que "eso es cosa de las abuelitas y abuelitos" prefieren dedicar su tiempo a otro tipo de actividades, ya que eso "pasó de moda" y es "anticuado". No obstante, no todos los jóvenes entrevistados comparten la misma opinión, generalmente quienes no están tan ligados a la tecnología manifiestan que la religión es parte indispensable de su identidad, es desde este espacio donde se fortalecen sus valores, encuentran paz y las respuestas a sus inquietudes. Lo anterior coincide con lo que expresa Rivas y Linarte (2015), Camarena y Tunal (2009), en relación a que a cada ser humano se le inculca desde la familia, cada individuo tiene la capacidad de ver el mundo y concebir la religión como un ente en el que puede refugiarse y desde la afirmación de que cada individuo influye en la construcción del otro.

De igual manera, hoy en día el acceso a la educación formal es una bendición para los jóvenes en la actualidad, antes la juventud se dedicaba a trabajar porque no había oportunidades de estudiar como ahora. Las escuelas son donde refuerzan los valores que se inculcan en el hogar, se aprenden habilidades para la vida y se les enseñan cosas que en la casa el papá o la mamá no pueden.

Sin embargo, también manifiestan que al sector educativo debería ser más exigente, incorporar materias que aborden contenidos culturales y de valores. Lo anterior se relaciona con lo que nuestros autores citados Maltos y Gell (2011) y Alemán y Díaz (2012), enfatizan sobre la importancia de la educación, la escolarización en la formación de la identidad. La escuela se puede ver como un estímulo para el desarrollo de la personalidad debido a que esta es homogeneizadora en cierto grado al desarrollar un proyecto que plantea contenidos y coordenadas comunes y exige el cumplimiento de reglas que afectan a todos.
Por otro lado, los jóvenes entrevistados expresaron que son ellos quienes más utilizan la tecnología, internet y redes sociales, reconocen la dependencia que hay a estos medios, incluso para realizar trabajos o tareas usan solamente el internet para más facilidad y rapidez, sustituyendo los viejos hábitos de leer un libro o de otros métodos, la tecnología además facilita la comunicación y la información, pero que desde el punto de vista cultural esto les ha afectado ya que se han retomado elementos pertenecientes a otras culturas.

Padres y madres (2) de familia, en entrevista refirieron que:

El uso de estas herramientas tecnológicas, es bueno hasta cierto punto, pero que en la actualidad la juventud lo ha tomado como "un vicio" y se acostumbran a esto, y que está afectando en el aspecto personal y familia. (Entrevista a padres y madres de familia, 18 de abril de 2016).

En este sentido se concuerda con lo que Vega (2014), refiere en cuanto a la aculturación que hoy en día se da a través de las redes sociales, mostrando prácticas y modos de comportamiento que provienen de otras culturas fuera de nuestro país. Otro aspecto importante son los medios de comunicación, tanto los jóvenes, padres y madres de familia entrevistados, coinciden en que los medios de comunicación existentes actualmente, influyen en gran medida en la personalidad de los jóvenes, ya sea a través de la televisión por cable, la radio o el teléfono móvil conectado a internet, transmiten contenidos diversos que atrapan a los diferentes grupos de la sociedad. Este planteamiento coincide con Miele (1996), quien explica que los medios de comunicación influyen en nuestra cultura, controlando parte de la sociedad desde las grandes potencias del mundo.

Así mismo es evidente la generalización de ideas, necesidades, artículos tecnológicos, sistemas, políticas que nacen de una estrategia económica global, pero que tiene repercusiones sociales, influyendo en nuestras actividades cotidianas, se hace tan necesario ahora mantenerse conectado al mundo moderno para estar a la orden del día y así poder competir en los diferentes ámbitos de la esfera social. Lo que manifestaron los entrevistados se asimila a lo expresado por Sánchez (2012), el cual menciona que la globalización ha producido, nuevas realidades en el concierto internacional, así 
como transformaciones en las relaciones socio-culturales mundiales.

\section{Vivencias de identidad cultural en la juventud}

Un elemento esencial en la identidad de cada persona son los valores que se inculcan en la niñez desde el hogar; sin embargo, hoy en día en la generación de la juventud actual hay una crisis en la práctica de estos valores, sobre todo el respeto, hacia los padres, madres, las personas de la tercera edad, las costumbres, las tradiciones, celebraciones religiosas y familiares, así como normas que se imponen en el hogar; ahora, tanto adolescentes como jóvenes no acatan las reglas sobre los horarios de salida y llegada a la casa sin ningún tipo de permiso, autorización o justificación de su salida.

En el caso de una familia en la que sus integrantes pertenecen a la etnia miskitu, la abuela (materna) manifestó que ni el papá ni la mamá les enseñan a sus hijos e hijas la lengua miskitu, algo muy importante que no se hace y que cuando ya crecen y son jóvenes, adultos no saben hablar su lengua materna, un elemento que debería ser parte de su identidad, que a la vez es parte de preservar la cultura de su etnia.

Lo anterior evidencia la influencia que tiene el entorno y grupos dominantes en la práctica de nuestras costumbres, por encajar a menudo dejamos a un lado características muy propias, en este caso la lengua, lo que viene a causar una crisis en las prácticas culturales, se dejan de retomar hasta que se olvidan, esto representa una pérdida en el patrimonio de aquellos pueblos que por años han venido tratando de mantener y preservar su cultura. Los resultados antes expresados concuerdan con lo que autores como Blanco (2008), Díaz (2006) y Ramírez (2008) al referirse que las y los jóvenes no serán los mismos de una generación a otra, pues las realidades cambian debido a factores que están presentes e influyen en el comportamiento de estos.

Ninguno se identificó con algún grupo de danza, escritura, poesía, pintura, conjunto musical; en nuestro municipio no hay grupos consolidados que desarrollen este tipo de actividades. Por su parte los jóvenes manifiestan que la manera en la que visten y se ven es una manera de expresar su identidad, quienes son y lo que les gusta, es lo que está a la moda, es actual, moderno y bonito.
La música que escuchan en la actualidad es música sin sentido "música de locos", que no tienen ningún mensaje sólo "loqueras" que influye negativamente en la juventud, porque les insta a consumir drogar y tener sexo irresponsable. Además, en las discotecas y bares no suenan música como la de antes (baladas, boleros, románticas, clásicas, Kawibe, Tipi, Dimensión Costeña), sólo suena un tipo de género musical.

Tampoco tienen el sentido de pertinencia hacia el entorno cultural que les rodea, no se identifican con los valores, costumbres y tradiciones de nuestra región. Para las personas adultas esto representa una preocupación porque si no se trabaja en ello, muchas riquezas, elementos autóctonos van dejándose de practicar, quedando en el olvido. Es bonito cuando un pueblo conserva sus costumbres, sus tradiciones, sus raíces y eso es símbolo de orgullo para las personas que lo conforman.

\section{Fortalecimiento de la identidad cultural de la juventud}

Tanto padres, madres y jóvenes, coinciden en que es importante tomar acciones desde la familia, ya que la base de la formación de la identidad y la personalidad de una persona se da desde el hogar a través de sus progenitores. La primera educación que se recibe en este ambiente tan esencial para el desarrollo de los jóvenes y todo ser humano.

La educación desde el seno familiar se manifiesta inculcando valores como el respeto, la responsabilidad, la honestidad, el amor al trabajo, a la vida. Lo anterior expuesto concuerda con lo planteado con Gómez y Suárez (2006), que resaltan que la familia y los adultos como seres significativos tienen gran impacto en la modelación del comportamiento del niño, el adolescente y el joven, a partir del ejemplo y la experiencia en la interacción diaria.

Además, desde el sistema educativo se debería implementar estrategias y metodologías efectivas que contribuyan en la formación de una identidad sana de adolescentes y jóvenes. Aprovechar espacios como el de Consejería Escolar. Esto concuerda con lo planteado por Infante y Hernández (2011), quienes manifiestan que la educación constituye una vía eficaz para conservar y desarrollar la identidad.

De igual manera, las instituciones o las organizaciones deberían de implementar actividades a 
desarrollar con la juventud que sean sanas y recreativas para que se distraigan de manera que puedan aprender valores que les ayuden en el transcurso de sus vidas. Porque a menudo los jóvenes no tienen a qué más dedicarse, además de ir al colegio. Esto se relaciona con lo planteado por Coffigny (2011), sobre la recreación como una manera de fomentar actividades con enfoque culturales.

\section{Conclusiones}

En general los jóvenes (mujeres y hombres) de ambos barrios, entrevistados, se identifican con elementos pertenecientes a la cultura mestiza, su idioma es el español, pero les atrae la idea de saber inglés o saber otro idioma que en el ámbito mundial sea importante y reconocido; asimismo, se identifican con la doctrina católica, aunque poco se retomen las prácticas religiosas hoy en día.

Los factores que inciden en la formación de la identidad cultural de los jóvenes, constituyen una serie de componentes clasificados en sociales, culturales, tecnológicos y económicos. Siendo el predomínate el tecnológico, debido al avance de la tecnología en el área de la información y la comunicación.

Las vivencias de identidad cultural en la juventud resultan un proceso enriquecedor debido a que cada persona expresa su forma de ser de acuerdo al contexto y realidad que le toca vivir. Sin embargo, las vivencias de los jóvenes no muestran una apropiación de los elementos culturales que caracterizan a nuestro territorio. Entre las acciones que se deben tomar para contribuir al fortalecimiento de la identidad cultural en la juventud están el promover espacios en la familia, la escuela, espacios públicos donde los jóvenes conozcan acerca de la cultura propia.

En definitiva, para contribuir al mejoramiento de la identidad cultural se proponen las acciones siguientes: a instituciones y organizaciones locales como promover actividades y concursos constantemente, de literatura, canción, danza teatro, el deporte, entre otros, para motivar a la juventud a interesarse por encontrar y desarrollar sus propias habilidades; a padres y madres de familia, fomentar en los jóvenes el interés por la cultura propia mediante la transmisión de conocimientos y saberes, a partir de la comunicación, el tiempo compartido en familia; y a la juventud, conocer la cultura propia, las tradiciones, costumbres que son originarias de la región y el significado que tienen. Involucrarse en las actividades de recreación cultural. Conversar con personas que sepan acerca de la historia de nuestra región y del municipio.

\section{Bibliografía}

Alemán, P., A., \& Díaz, T. (2012). La educación como factor de desarrollo. Recuperado de: http:// www.monografias.com/trabajos48/educacion-y-desarrollo/educacion-y-desarrollo2. shtml

Blanco, J., A. (2008). Usos, Consumos y atributos que los jóvenes Guanajuatenses otorgan a las tecnologías de información y comunicación. Recuperado de: http://www.eumed.net/tesis-doctorales.

Camarena, M. E., \& Tunal, G. (2009). La religión como una dimensión de la cultura. Recuperado de: pendientedemigracion.ucm.es/info/nomadas/22/tunal_camarena.pdf

Coffigny M., Y. (2011). Actividades recreativas deportivas para el desarrollo de los adolescentes de 12 años. Recuperado de: http://www.monografias.com/

De la Hoz, F. (2011). El desempleo juvenil: problema de efectos perpetuos. Recuperado de file:///C:/ Users/Admin/Downloads/616-1517-1-PB.pdf

Díaz, J. (2006). Identidad, adolescencia y cultura. Recuperado de: http://evaluaciondocente. sep.gob.mx

Gómez, C. (2009). La familia: Pre- modernidad, Modernidad. Recuperado de vinculologia. blogspot.com/.../la-familia-premodernidad modernidad.htm.

Gómez, L., \& Suárez, O. (2006). Habilidades para la vida. Recuperado de: http://www.udea.edu. $\mathrm{co} /$

Guerrero, P.(2002). GuíaEtnográfica; Sistematización de datos sobre la diversidad y la diferencia de las culturas. Quito, Ecuador. Abya- Yala.

Infante, M., E., \& Hernández, R., C. (2011). Preservar la identidad cultural una necesidad en la actualidad. Recuperado de: http://asri. eumed.net/o/imhi.html 
Instituto Nicaragüense de Cultura (2012). Nuestros patrimonios...Nuestro Bienestar. Recuperado de: http://www.inc.gob.ni/index.php?

JENH - CEDEHCA. (2007). Agenda de adolescentes $y$ jóvenes Afrodescendientes de la Costa Caribe de Nicaragua. Nicaragua. Unicef.

León, A. (2013). Identidad Cultural. Recuperado de: https://arturoleonb.wordpress. com/2013/o4/o1/identidad-cultural/

Maltos, M., y Gell, A. (2011). Papel de la familia en el desarrollo de la identidad. Recuperado de: http://www.eumed.net/rev/cccss/13/magl. html

Miele, M. (1996). Identidades Urbanas. Ecuador: UPS.

Ramírez, M., \& Mazariegos, L. M. (1993). Tradición y modernidad: lecturas sobre la cultura maya actual (No. 3).Guatemala. Universidad Rafael Landívar, Instituto de Lingüística.

Rascón, M., T. (2006). La construcción de la identidad cultural. Recuperado de: www.the.uma.es/ personal/ver/id/23
Rivas, D., y Linarte, J. (2015). Factores psicosociales que influyen en el proceso de construcción de la identidad personal en Estudiantes de la carrera de psicología. Recuperado de: http://es.slideshare.net/DonaldRivas/identidad-personal-factores-psicosociales?from_action=save

Sánchez, M (2012). Identidad cultural latinoamericana desde la perspectiva de José Martí. Recuperado de www.eumed.net/rev/ccss/2o/ sgdb.html

Sandin, M. (2003). Investigación cualitativa en investigación. Fundamentos y Tradiciones. Madrid: McGraw-Hill.

Universidad Centroamericana [UCA], (2013). La familia nicaragüense en proceso de cambio. Envío. (34). Recuperado de: http://www.envio.org. ni/articulo/419

Vega, R. (2014). Identidad Cultural de los pueblos indígenas. Recuperado de: biblio3.url.edu.gt/ Tesario/2014/o7/o1/.pdf 